\title{
Assessment of anthropometric measurements and body composition of selected beginner South West Ethiopian soccer players
}

\author{
Esayas HAILU ${ }^{1}$, Daniel KIBRET², Abera TOMAY ${ }^{1}$ \\ ${ }^{1}$ Department of Sport Science, Mizan-Teppi University, Ethiopia. \\ 2 Department of Sport Science, DebreMarkos University Ethiopia \\ Address Correspondence to E.Hailu, e-mail: gudu888@gmail.com
}

\begin{abstract}
This study attempts to determine the anthropometric measurement and body composition quality of south west Ethiopian beginner soccer players' considering playing position. In so doing, three soccer teams were selected by employing a cluster sampling techniques that consisted of a total forty eight players, and those who attended less than seventeen years of age. Depending on their playing position players were classified in to four categories as goalkeeper (GK), defense (DF), midfielder (MF) and striker (SK). To achieve the stated purpose, cross sectional descriptive research design was employed. The international Society for the Advancement of Kinanthropometry (ISAK) protocol was considered to measure the following anthropometric variables :weight measurements, $\mathrm{n} \_1$; girths, $\mathrm{n} \_/ 10$; lengths, $\mathrm{n} \_/ 6$, skin folds, $\mathrm{n} \_/ 2$; (body fat $\%$ and lean mass)and body mass index $\mathrm{n}-1$.The data was analyzed by SPSS version 19 , moreover, the level of significance was set at $(\mathrm{P} \leq 0.05)$. The finding of this study depicted that significant difference of anthropometric measurement among players based on their playing position. DF players possess larger lower limp, interior body and upper limp girth. Whereas, SK player exhibited smaller lower limp, interior body and upper limp girth. In lower limp length GK and SK have larger lower limp length but in upper limp GK possess greater. GK $(176.6 \mathrm{~cm})$ are taller than all other players of team while MF $(170 \mathrm{~cm})$ is sorter in overall body height. There is no significant difference in BMI(GK 19, DF 20.4, MD 18 and SK 18), lean mass and body fat\% But GK(64.2 kg) are heavier while SK (58kg) lighter in their weight. Whereas, DF $(60.7 \mathrm{~kg})$ and MF $(60 \mathrm{~kg})$ players are average in their weight. It is found that mean anthropometric measurement of south west Ethiopian youth soccer players was slightly lower than that of top world class players of similar age group players. It is also obtained that lack of significant differences among playing positions reflect on BMI, lean mass and body fat $\%$ indicate as coaches are not give playing position specific training for players. In this study, within-position variation was quite large in some cases, which could indicate that a team that does not have the opportunity to hand-pick players, based on anthropometric characteristics, may be at a disadvantage therefore the respective soccer coaches, sport science professionals should take into account the principle of morphological optimization in talent detection, identification and selecting soccer players.
\end{abstract}

Keywords: Anthropometry, body composition, soccer.

\section{INTRODUCTION}

Soccer one of the most popular team sport which is characterized by high intensity, short-term actions and pauses of varying length (26). To succeed soccer game, players need the optimal combination of physical quality, technical, tactical, and mental motivation (4). Due to the financial benefits of being able to promote talented players from the youth ranks into the senior first team, sports scientist employed crucial role on a professional soccer club. They in identify and develop future players. Information concerning the anthropometric and performance characteristics of players of varying age will have application to a large population, particularly coaches and sports scientists (22).

Anthropometry is the branch of anthropology that is worried about human body measurement. The definition has confined to the kind of measurements commonly used in associating physical performance with body build. Anthropometry involves the 
measurement of external part of the body, including body diameters, body circumference, heights and breadth (11).Indeed, many experts in the field, such as soccer coaches, managers and scientists believe that the success of this sport can be associated with anthropometric characteristics of players. Even, some studies have focused on the relationship between anthropometric profiles of players and their standard positions (12).

Anthropometric quality of soccer players is a major determinant of their success in playing genuine soccer however it associates within a playing position. For example a taller player is most suitable for central defensive positions, goalkeeping, and central attack $(21,22)$. Not only these, specific anthropometric characteristics needed to be successful in certain sporting events. It is also important to note that there are some differences in body structure and composition of sports persons involved in different sporting event. The process whereby the physical demands of a sport lead to selection of body types best suited to that sport is known as "morphological optimization" (6).

In soccer, the importance of body composition on performance remains unclear; however, it is a Primary concern in conditioning programs throughout a season at all levels of competition. But body composition measures are widely used to prescribe desirable body weights, to optimize competitive performance, and to assess the effects of training (24). A lower relative body fat is desirable for successful competition in most of ball games. This is because additional body fat adds to the weight of the body without contributing to its force production or energy producing capabilities, which means a decrease in relative strength $(1,24)$.

In measuring this aspect of body composition, the total body weight is divided into two components; Lean Body Weight and Fat Body Weight. Lean Body Weight includes muscle, bone and vital organs and estimated from skin fold measurement (27).

Physical characteristics and body composition have been known to be fundamental to excellence in athletic performance (16). Specific athletic events require different body types and weights for maximal performance (2). Today it has been widely accepted by the experts that top performance in soccer is achieved if player possesses the basic body composition and anthropometric characteristics suitable for his/her position(goalkeeper defense, midfielder and striker) (15). At present, sportsman for superior performance in any sports is selected on the basis of physical structure and body size.

Developing good conditioning programs based on the specific morphological and physiological requirement of each sport is considered as a key factor for successes (3). Actually both anthropometry and body composition have relationship with soccer performance. But still more clarification is required on anthropometric and body composition qualities Ethiopian beginner soccer players. So this research was conduct to fill this gap. It is an interesting topic for physical exercise scientists, coaches, athletes, exercise physiologist and other specialists in sports and exercise science.

In fact, there were many studies regarding to analyzing the exercise and fitness, but there were no enough researches that studied the anthropometric and body composition quality and requirements of soccer game, especially in Ethiopia in relation to this issue.

Currently, enhancement of athletic performance efficiency is designed upon critical study of human anatomy, physiology, modern way of feeding and scientific way of training based upon a new findings and principles of investigation. This study was highly concentrated on the assessment of anthropometric measurement and body composition of beginner South West Ethiopia Soccer players, in relation with their playing position but it does not mean that the outcome of this research is restricted to South west Ethiopia.

The research will contribute in addressing the anthropometric and body composition characteristics of soccer players for coaches, players and managers to understand, formulate and implement on designing effective strategies for coaching program. The study also helps to develop our country's soccer federation to recruit skill full players based on their anthropometric quality. In addition, it will help for others as a research work for depth studies on the problem under taken. The finding of the research may help as reference for researchers who will conduct advanced researches. 
The general objective of this study was to determine anthropometric and Body composition quality of South west Ethiopia beginner soccer players in relation with their playing position.To examine the anthropometric quality of south west Ethiopian, beginner soccer players based on their playing position. To assess body composition of school level soccer players of south west Ethiopian in relation with their playing position.

\section{MATERIAL \& METHOD}

The source of population for this study was male, youths of BenchiMaji, Kaffa and Shaka Zones; that was representing their zones in 2015 South Nation, Nationality and Peoples region School soccer tournament.Male, healthy, Schools soccer players of BenchiMaji, Kaffa and Shaka Zones and those who attended less than seventeen years of age was the subjects of the study. This research was conducted at Mizan, Bonga and Masha Towns. The study was applied for three months; beginning from July 2015 to September 2015.

The researchers collectedthe primary data, from body mass index measurements, three site, four site skin fold measure, heights includes; standing height, sitting height, arm, fore arm and leg and girths includes; fore arm, thigh, calf, waist, and chest, neck, wrist and ankle and body weight. Secondary data was used from journals, books and magazines relevant to the research.

Descriptive research design was used for this study; the detail operation is led by cross-sectional method. The research was conduct with forty eight (48) under seventeen year's young male soccer players from Kaffa, Bench Maji and Shaka district (south west Ethiopia). Beside this, the research was focused on anthropometric and body composition quality of best soccer players who was selected from different towns to represent their zones in relation with their playing position.

This research study was carryout and governed by the regulations for research on human beings. To this fact, the privacy of the participants was protected, Permission was obtain from authorized administrator of zones sport office and signed consent was provide to participant earlier with a written letter. The ethical considerations was include; all of the participant have clear information about the purpose of the study, the procedure to be used, the potential benefit and possible risk of participation in this study. As well as, result was keep confidentially. Any type of information would not disclose to anyone except the researchers and the assistance technician in this experiment.

\section{Sample and sampling techniques}

The cluster sampling technique was used to select three soccer teams, which consists sixteen up to twenty five beginner soccer players. Their age was less than seventeen years. The sample size for this study was forty eight. First Permission was obtained from authorized administrator of zone sport office. Then all players was request to fill medical history questionnaire, which prepared with the aim of identifying whether they are free from acute and chronic sport injury and any anatomical impairment.

\section{Variables}

Height; Standing, sitting, arm, fore arm, medial and lateral tibiae, Girth; ankle, calf, thigh, hip, waist, chest, arm, fore arm, neck and wrist, body compassion; three site, four site skin fold measurement and body mass index and body weight.

\section{Anthropometric measures}

On the day of testing, the standing and sitting height, upper limb height (forearm and arm), lower limb height (tibiae medial and tibiae lateral), and body mass was determined using a standard weighing machine, stadiometer, calibrated and large sliding caliper. For accuracy researcher was use the manufacturer's guidelines. The height and weight of subjects was measure to the nearest $0.5 \mathrm{~cm}$ and $0.1 \mathrm{~kg}$, and obtained with the participants in athletic shorts, shirt and bare feet.

Girth or circumference measurements was token according to previously described and validated methods (8) protocol using a self-retracting, inelastic, nonmetallic anthropometric measuring tape. If swelling was present, the measurement also excluded. Two technicians performed a single measurement at each point then these values were averaged for further calculations.

\section{Body composition}

Four-site and three site skin fold measurements(14)were taken following techniques described by Harrison et al.(13)and using standard 
calibrated skin fold calipers, which maintained constant pressure. The caliper was held in the right hand, the skin fold elevated with the left hand, and the measurement recorded four seconds after pressure was release.

Three site Skin fold fat was obtained at the chest, triceps, sub scapular locations in accordance with previously accepted procedures Jackson AS, Pollock ML, $(14,20)$ whereas the Four site skin fold fat was obtained by Ross craft Calipers (British Indicators, UK) at four sites (biceps, triceps, subscapular and suprailiac) as recommended by(9). Two technicians was performed a measurement at each site; then two measurements at each site was recorded and later averaged for further calculations. The body mass index $\left(\mathrm{kg} / \mathrm{m}^{2}\right)$ was calculated for each subject. Body weight classification for subjects was determined as described by (7).

\section{Protocol}

Using ISAK accredited methods; a total profile of 24 measurements was collected from each player. All data collection was performed by an ISAK protocol. The players were informed about the measurements to be taken, and the different positions required for measurement were explained and demonstrated before the start, to ensure that the procedure was quick and efficient.

All players were measured in a private consulting area to ensure privacy during data collection. The measurements were taken at temperature was held constant at or around $22^{\circ} \mathrm{C}$. They wore minimal clothing (training shorts and bare chest) to allow access to all measurement sites. Where possible, a recorder was present to assist the anthropometries and enter data into a software program. The equipment used for taking measurements included a stadiometer (Lester, UK), electronic weighing scales (SECA, UK), a small sliding caliper (Rosscaft, Canada), a large sliding caliper, and skinfold caliper (Harpenden@ British Indicators Ltd., Luton,UK). A complete data set was obtained before repeating the measurements for a second time to help minimize the effects of skin compressibility. All measurements were taken in the same order and, to avoid exercise-induced hypertrophy of muscles, all players refrained from exercise for at least 1 hour before the measurement session.
The researchers were collecting the data with the help of three trained assistant technicians (BSc holders). To avoid errors, five day training was given for the assistance data collector on how to use data collecting instruments and measurements during data collection. Only standardized materials were used to keep the quality of the data. Additionally, all the aforementioned tests was record with video. Finally, the data had been coded and feed to software twice, with different persons to avoid error in data feeding.

\section{Data Analysis}

The data analysis was done by SPSS statistical software package Version 19. After the data was collect on weight, height, girth and body composition it was analyzed by descriptive statics. Moreover, the level of significance was set at $(\mathrm{P} \leq 0.05)$.

\section{RESULTS \& DISCUSSION}

\section{Overview}

In this study, Anthropometric assessment had been taken on three major categories (girth, height and weight) and body composition has been taken from skin fold measurement and body mass index. Only players with full data sets were used in the statistical analysis (SPSS, Version 19.0). Means and standard deviations $(\mathrm{M} \pm \mathrm{SD})$ were calculated for further interpretation.There is no significant difference on the age of all players based on their playing position $(\mathrm{P}>0.21)$, the reason is that as all of the subject are student at secondary and preparatory class.

Table 1. Age of subject.

\begin{tabular}{llcc}
\hline No & Players & Number & Age mean + SD \\
\hline 1 & Goalkeeper & 6 & $16.5 \pm 0.43$ \\
2 & Defensive & 18 & $15.5 \pm 0.83$ \\
3 & Mid fielder & 18 & $16.5 \pm 0.40$ \\
4 & Strikers & 6 & $16.1 \pm 0.98$ \\
\hline
\end{tabular}

\section{Anthropometric variables}

Results were presented as means and standard deviation in tables. Mean anthropometric values of lower limp girth measurement of GK $23.1 \pm 3.1 \mathrm{~cm}$, $34 \pm 2.7 \mathrm{~cm}, \quad 84.5 \pm 5.3 \mathrm{~cm}, \quad$ DF $24.6 \pm 2.7,36 \pm 3.3 \mathrm{~cm}$, $87.45 \pm 4.3 \mathrm{~cm}$, MF $23.5 \pm 2.6 \mathrm{~cm}, 35.5 \pm 2.4 \mathrm{~cm} 85.5 \pm 5.3 \mathrm{~cm}$, SK $22.2 \pm 1.3 \mathrm{~cm}, 35.0 \pm 1.4 \mathrm{~cm}, 84.5 \pm 5.3 \mathrm{~cm}$ of ankle, calf and thigh circumference respectively. This mean 
result indicate that as defensive players of this three team have larger lower limp circumference than the rest of players which allow them to resist combat with the opponent and equipment. Statistically significant difference was noted in the calf girth measurement among players $(p=0.04)$. Whereas the goalkeepers reveal slim lower limp relative to their team mate.

In hip, waist and chest girth measurement defensive players were exhibited larger body structure as we realize from the mean results. The mean result for hip, waist and chest of each categories are listed respectively as follow GK $88.7 \pm 6.2 \mathrm{~cm}, 74.3 \pm 8 \mathrm{~cm}, 87.5 \pm 7.2$ DF $91.9 \pm 5.5 \mathrm{~cm}, 79.9$ $\pm 6.7 \mathrm{~cm}, 92.3 \pm 6.3 \mathrm{MF} 89.6 \pm 6.8 \mathrm{~cm}, 76 \pm 7.5 \mathrm{~cm}, 89.0 \pm$ 7.6 SK $87.1 \pm 2.6 \mathrm{~cm}, 68.5 \pm 1.3 \mathrm{~cm}, 79.5 \pm 1.6 \mathrm{~cm}$. Statistically significant difference was noted in the all hip, waist and chest circumference $(\mathrm{p}<0.05)$. But the strikers have very trim upper body structure relative to other players, which makes them disadvantageous in playing soccer.
In upper limp circumference, the same to lower body girth defensive player comprise larger upper limp structure. Mean \pm SD result of wrist, arm and forearm of players are state as follow in their respective order GK $17.4 \pm 16 \mathrm{~cm}, 27.1 \pm 4.2 \mathrm{~cm}, 24.3 \pm$ $4.2 \mathrm{~cm} \mathrm{DF} 17.5 \pm 1.3 \mathrm{~cm}, 29.5 \pm 3.1 \mathrm{~cm} 27 \pm 2.8 \mathrm{~cm}$, MD17.5 $\pm \quad 1 \mathrm{~cm}, 28.5 \pm \quad 3.9 \mathrm{~cm} \quad 25.5 \pm \quad 3.7 \mathrm{~cm} \quad$ SK16.4 \pm $0.8 \mathrm{~cm}, 24.2 \pm 1.4 \mathrm{~cm} 24 \pm 0.6 \mathrm{~cm}$. We observed from the above mean result the striker players are possess thin upper limp where as defensive players are thick upper limp in respect to rest subject.

Overall there is heterogeneity on result of circumference among the subjects but significant dominancy is observed by defensive players in thickness of whole body structure whereas in case strikes the inverse is true.

Table 2. Lower limp and anterior body girth measurement of the subjects (cm).

\begin{tabular}{llcccc}
\hline Variables & & GK & DF & MF & SK \\
\hline Ankle & Mean \pm SD & $23.1 \pm 3.1$ & $24.6 \pm 2.7$ & $23.5 \pm 2.6$ & $22.2 \pm 1.3$ \\
& MD & +1.1 & +2.4 & +1.3 & -2.4 \\
Calf & Mean \pm SD & $34.3 \pm 2.7$ & $36.0 \pm 3.3$ & $35.5 \pm 2.4$ & $35 \pm 1.4$ \\
& MD & -1.2 & +1.7 & +1.2 & +.7 \\
\multirow{5}{*}{ Thigh } & Mean \pm SD & $84.5 \pm 5.3$ & $87.4 \pm 4.3$ & $85.5 \pm 5.3$ & $84.6 \pm 4.8$ \\
& MD & -3 & +3 & +1 & +.01 \\
Hip & No & 6 & 18 & 18 & 6 \\
& Mean \pm SD & $88.7 \pm 6.2$ & $91.9 \pm 5.5$ & $89.6 \pm 6.8$ & $87.1 \pm 2.6$ \\
Waist & MD & +1.6 & +4.8 & +2.5 & -4.8 \\
& Mean \pm SD & $74.3 \pm 8$ & $79.9 \pm 6.7$ & $76.0 \pm 7.5$ & $68.5 \pm 1.3$ \\
Chest & MD & +5.6 & +11.4 & +3.9 & -11.4 \\
& Mean \pm SD & $87.5 \pm 7.2$ & $92.3 \pm 6.3$ & $89.0 \pm 7.6$ & $79.5 \pm 1.6$ \\
& MD & +8 & +12.8 & +9.3 & -12.8 \\
\hline From the above & table, mean \pm SD are value of ankle, calf, thigh, hip, waist, chest. GK= goalkeeper, & 6 \\
MF=midfielderender
\end{tabular}

Table 3. Upper limp girth measurement of the subjects $(\mathrm{cm})$.

\begin{tabular}{llcccc}
\hline Kind & & GK & DF & MF & SK \\
\hline Wrist & Mean \pm SD & $17.4 \pm 1.6$ & $17.5 \pm 1.3$ & $17.5 \pm 1$ & $16.4 \pm 0.08$ \\
& MD & +1 & +1.1 & +1.1 & -1.1 \\
Arm & Mean \pm SD & $27.1 \pm 4.2$ & $29.5 \pm 3.2$ & $28.5 \pm 3.9$ & $24.2 \pm 1.4$ \\
& MD & +2.6 & +5.3 & +4.3 & -5.3 \\
Fore arm & Mean \pm SD & $24.3 \pm 4.2$ & $27.08 \pm 2.8$ & $25.5 \pm 3.7$ & $24.0 \pm 0.63$ \\
& MD & +0.3 & +3 & +1 & -3 \\
\hline
\end{tabular}

From the above table, mean $\pm \mathrm{SD}$ are value of wrist, arm, fore arm girth.GK= goalkeeper, $\mathrm{DF}=$ defender $\mathrm{MF}=$ midfielder $\mathrm{SK}=$ striker players, $\mathrm{MD}=$ mean difference. 
Table 4. Upper limp, lower limp and height measurement of the subjects (cm).

\begin{tabular}{llcccc}
\hline Kind & & GK & DF & MF & SK \\
TM & Mean \pm SD & $44.6 \pm 2.6$ & $41.8 \pm 1.9$ & $44.4 \pm 12.7$ & $45.8 \pm 1.4$ \\
& MD & +2.8 & -4 & +2.6 & +4 \\
TL & Mean \pm SD & $51.3 \pm 4.4$ & $48.0 \pm 5.0$ & $46.2 \pm 4.5$ & $48.5 \pm 3.0$ \\
& MD & +5.1 & +1.8 & -5.1 & 2.3 \\
AR & Mean \pm SD & $35.5 \pm 2.6$ & $33.3 \pm 2.8$ & $34.3 \pm 1.5$ & $35.5 \pm 0.54$ \\
& MD & +2.2 & -2.2 & +1.0 & +2.2 \\
& No & 6 & 18 & 18 & 6 \\
FAR & Mean \pm SD & $28.6 \pm 0.8$ & $27.5 \pm 1.6$ & $27.1 \pm 1.6$ & $27.8 \pm 1.4$ \\
& MD & +1.5 & +0.4 & -1.5 & +0.7 \\
SH & Mean \pm SD & $85.1 \pm 5.4$ & $88.0 \pm 4.6$ & $85.0 \pm 2.9$ & $87.5 \pm 1.04$ \\
& MD & +2.9 & +3 & -3 & +2.5 \\
StH & Mean \pm SD & $176.6 \pm 6.1$ & $172.6 \pm 7.7$ & $170.0 \pm 6.1$ & $170.8 \pm 5.1$ \\
& MD & +6.6 & 2.6 & -6.6 & +0.8 \\
& No & 6 & 18 & 18 & 6 \\
\hline \multicolumn{2}{l}{ From the above table mean \pm SD are value of Tibia lateral (TL), tibia medial(TM), arm (AR), fore arm(FAR), siting height(SH), } \\
standing height(StH) height. GK= goalkeeper, DF=defender MF=midfielder SK= striker players, MD=mean difference.
\end{tabular}

The height measurements were obtained from subjects' arm, fore arm, sitting height and standing height. The mean result indicate as there is significant heterogeneity based on their playing position. This heterogeneity of Tibia medial, tibia lateral, arm length and fore arm length of player are stated as follow in respect to their order as mean \pm SD, GK $48.6 \pm$ $2.6 \mathrm{~cm}, 51.3 \pm 4.4 \mathrm{~cm}, 35.5 \pm 2.6 \mathrm{~cm}, 28.6 \pm 0.8 \mathrm{~cm}, \mathrm{DF} 41.8 \pm$ $1.9 \mathrm{~cm}, 48.0 \pm 0.5 \mathrm{~cm}, 33.3 \pm 2.8 \mathrm{~cm}, 27.5 \pm 1.6, \mathrm{MF} 44.4 \pm$ $12.7 \mathrm{~cm}, 46.2 \pm 4.5 \mathrm{~cm}, 34.3 \pm 1.5 \mathrm{~cm}, 27.1 \pm 1.6$, SK $49.0 \pm$ $1.4 \mathrm{~cm}, 8.5 \pm 3 \mathrm{~cm}, 35.5 \pm 0.54 \mathrm{~cm}, 27.8 \pm 1.4 \mathrm{~cm}$ as the above mean result reveal goalkeepers and striker players have longer lower limp length than rest of the players. This makes them advantages in increasing stride length during moment of game, which is essential to cover large distance with few gaits'. In upper limp, goal keepers have significantly higher length than others. This helps them to handle the ball during goal keeping.

The sitting height and standing height of the subjects are explore as follow according to their order, GK $85.1 \pm 5.4 \mathrm{~cm}, 176.6 \pm 6.1$, DF $88 \pm 4.6 \mathrm{~cm}, 172.6 \pm$ $7.7 \mathrm{~cm}, \mathrm{MD} 85 \pm 2.9 \mathrm{~cm}, 170.0 \pm 6.1$, SK $87.5 \pm 1.04 \mathrm{~cm}$, $170.8 \pm 5.1$ as we understand from above mean result goal keeper players where taller than the rest of players. These characteristics would help GKs in aerial duels allowing them to defend their goals. Relatively strikers are shorter from their team mates $(\mathrm{P}<0.05)$.

The heterogeneity in height of players is confined with the study conduct in Tunisia before two years in 2013 by Mehdi (19), which entitled Anthropometric and Physical Characteristics of Tunisians Young
Soccer Players. He point out as goal keeper players were taller and heavier than other group of soccer players.

The results of the present study also showed significant differences between playing position standards concerning the anthropometric measures, especially body weight and height. This result is in agreement with Gill et al.(12) that GKs were heavier and taller than other playing position groups. Other anthropometric differences according to playing position were identified. Our results are consistent in partly with the findings of Malinaet al. (17) who conduct in soccer players aged 11 to 16 years old, that point out FWs were taller than DFs and GKs were heavier than MFs. Our data are similar to those reported by Slavko et al. (23) in amateur German football players but different to those reported by Wong and et al.(28) in Under 14 years soccer players. This discrepancy could be explained in part by the sample size, the different methods of measurement as well as the performance level. The results of the current study showed substantial variations in stature and body mass suggesting that there are different physical demands in each playing position standard.

\section{Body composition variable}

The goalkeepers were also significantly heavier than the midfielders and striker. Despite a mean difference of $5.5 \mathrm{~kg}$, differences between the midfielders and defensive players were no statistically significant. The defenders were significantly heavier than the strikers, but did not differ significantly from the midfielders. Research suggests that midfielders have a lighter body mass to move through space more 
efficiently, enabling them to cover greater distances. Defenders tend to be heavier and taller with less body fat, as their position requires them to be robust and strong in the tackle (5).

The breakdown of body compartments for the subjects on their different playing positions is shown in Table 5. There were no statically significant differences in BMI or lean mass between the different positions, but defensive players were exhibit higher body BMI whereas strikers are the lowest than the rest of players. The percent body fat analysis revealed that the differences occurred between the defensive players and each of the outfield groups, with no statically significant differences evident between rest playing positions.

The heterogeneity in body compartment of players depending on their playing position was tolerable and acceptable in soccer game. The present study also insures this quality of soccer players. The finding of this study is partially agree with finding of Sutton et al. (25), entitled "Body composition of English Premier League soccer players: Influence of playing position, international status, and ethnicity" their result explore that goal keepers are heavier than the rest of players by their body mass and their lean mass almost similar to rest of defender, midfielders and strikers. But if we can compare the men result of English Premier League players and south west Ethiopian soccer players, Ethiopian south west players GK $64.2 \mathrm{Kg}$, DF 60.7, $\mathrm{Kg}, \mathrm{MF} 60 \mathrm{Kg}$ and SK $58 \mathrm{Kg}$ whereas English Premier League players GK 90Kg, DF86Kg, MF 78Kg SK82.7. Despite age variation between this two groups there are mean of $23 \mathrm{~kg}$ body mass variation.

This study is also partially consistent with study conducted by Mark Russell \& Edward(18). Tooley on UK before three years under title "Anthropometric and performance characteristics of young male soccer players competing in the UK" but the mean result on body mass have variation of $11 \mathrm{~kg}$ with this study. We conclude that south west Ethiopian soccer players have similar Lean mass and BMI but less in their body mass from rest of world beginner players.

Our finding is concurring with those of previous studies that focused on the anthropometric characteristics of elite soccer teams. Reilly et al.(22) found that relative heterogeneity in body size is a characteristic of elite soccer teams, so anthropometric differences were therefore expected between playing positions. Previous studies have reported significant differences in a variety of anthropometric characteristics, most notably stature and body mass, perhaps suggesting that these variables denote a morphological optimization within soccer.

In conclusion; based on the results of this study, the following conclusions drawn about, South west Ethiopian beginner soccer players.

- Generally, the goalkeepers are heavier and taller than other subject of the study but relatively strikers were posse's slim body and average in their height.

- Defensive players possess larger almost in all girth measurements. But this has no influence on their game performance since they are not exposed to cover large distance during the game.

- Lack of inter-positional differences among the players' body fat $\%$ lean mass $\%$ and BMI components could also imply that there is no position specific training in the team. All the players are given similar training prescription during practice.

- Anthropometries of south west Ethiopian soccer players are comparably lower than those of players elsewhere in the world.

Table 5. Body mass index, percent lean mass, and percent body fat of subjects grouped by their playing position.

\begin{tabular}{|c|c|c|c|c|c|c|}
\hline \multirow[t]{2}{*}{ No } & \multirow[t]{2}{*}{ Kind } & & \multicolumn{3}{|c|}{ Playing position } & \multirow[b]{2}{*}{ Striker } \\
\hline & & & Goalkeepers & Defenders & Midfield & \\
\hline & No. of players & & 6 & 18 & 18 & 6 \\
\hline & $\operatorname{Mass}(\mathrm{Kg})$ & Mean \pm SD & $64.2 \pm 4.6$ & $60.7 \pm 7.3$ & $60.0 \pm 5.8$ & $58.7 \pm 5.6$ \\
\hline & BMI & Mean \pm SD & $19.0 \pm 3.0$ & $20.4 \pm 1$ & $18.0 \pm 2.1$ & $18.0 \pm 1.8$ \\
\hline & Lean mass \% & Mean \pm SD & $79.9 \pm 1.2$ & $73.4 \pm 2.2$ & $81.1 \pm 1.9$ & $80.3 \pm 2.1$ \\
\hline & Body Fat $\%$ & Mean \pm SD & $12.9 \pm 2.0$ & $16.6 \pm 2.1$ & $10.2 \pm 1.8$ & $9.9 \pm 2.0$ \\
\hline
\end{tabular}

From the above table, mean \pm SD are value of mass, BMI=body mass index, lean mass $\%$ and body fat $\%$ of goalkeeper, defender midfielder and striker players 
Recommendation; based on these results, discussions and findings of the research, the following recommendation is made.

- Soccer coaches should be adopt a scientific approach to coaching and systematic Talent Identification and selection rather than relying on traditional non-scientific prognostic approaches.

- Ethiopian sport scientist, researchers, sport administrators and coaches has to be understand the role of anthropometry on soccer performance and give emphasis during talent selection, detection and recruiting players for team.

- As we Ethiopian nation are compute in soccer with the world, all concerned body has to be use anthropometric and body composition quality of top world class players as bench mark point for optimizing physical quality of our soccer players.

\section{REFERENCES}

1. Abraham G. Analysis of anthropometry, body composition and performance variables of young Indian athletes in Southern Region. Indian Journal of Science and Technology 2010; 3(12):1210-1213

2. American Dietetic Association. Position of the American Dietetic Association Nutrition for the physical fitness and athletic performance for adults. J. Am. Diet Assoc.1987; 76: 437443.

3. Balciunas MC. Hyperlink. Long Term Effects of Different Training Modalities on Power, Speed, Skill and Anaerobic Capacity in Young Male football Players.J. ofSpo. Scie. and Med.2006; 5:163 - 170 .

4. Bangsbo J, Michalsik L. 2002. Assessment and physiological Capacity of elite soccer players. In T. Reilly, \& A. Murphy (Eds.), Science and football IV (pp. 53-62). Cambridge, UK: Routledge,

5. Bell W, Rhodes G. The morphological characteristics of the association football player. Journal of Sports Medicine and Physical Fitness,1980: 20, 196_200.

6. Bloomfield J, Fricker Peter A, Fitch Kenneth D. Can running injuries be effectivelyprevented?j. Sci. Med. Sports. 1995: 1, 161.

7. BrayG. Obesity in American. Proceedings of the 2nd Forgaty International Center Conference on Obesity. Washington DC. NIH Publication, 1979: 79.

8. Callaway C, Chumlea W, Bouchard C. Anthropometric Standardization Reference Manual. In: Lohman, T.,Roche, A., Martorell, R. editors. Champaign, IL: Human Kinetics, 1988:3954.
9. Durnin J, Rahaman, M. The assessment of the amount of fat in the human from measurements of skinfold thickness. Br. J. Nutr. 1967; 21: 681-689.

10. Drust B, Cable NT, Reilly T. Investigation of the effects of the pre-cooling on the physiological responses to soccer-specific intermittent exercise. European Journal of Applied Physiology, 2000; 81, 11-17.

11. Findak V, Metikoš D, Mraković M, Neljak B. 1996. Primjenjenakine- ziologija u skolstvu NORME. Hrvatskipedagosko-knjizevnizbori F akultetzafizickukulturu SveucilistaZagrebu, Zagreb.

12. Gil S, M Gil J, Ruiz F, Irazusta A, Irazusta J.Physiolo- gical and anthropometric characteristics of young soccer player's according to their playing position: relevance for the selection process. Journal of Strength and Conditioning Research, 2007: 21, 438-445.

13. Harrison G, Buskirk R, Carter J. Skinfold thicknesses and measurement technique. In: Lohman and T., Roche, F. and Martorell, R. editors. Anthropometric Standardization Reference Manual. Champaign, IL: Human Kinetics, 1988: 5570

14. Jackson, A. Pollock M. Practical assessment of body composition. J. Phys Sport's med., 13(5):76-90.

15. Latimer- cheung MP, Krustrup J. Bangsbo. 2013. Match performance of high standard soccer players with special reference to development of fatigue. J. Spo. Scie.,1985; 21: 519528.

16. Mathur D, Salokun S. Body composition of successful Nigerian female athletes. J. Sports Med., 1985; 25: 27-21.

17. Malina RM, Pena Reyes ME, Eisenmann J C, Horta L, Rodrigues J, Miller R. Height, mass and skeletal maturity of elite Portuguese soccer players aged 11-16 years. Journal of Sports Science, 2000: 18, 685-693.

18. Russell M, Tooley E. Anthropometric and Performance Characteristics of Young Male Soccer Players Competing in the Uk. Serbian Journal of Sports Sciences, 2011; 5(4): 155-162

19. Mehdi B, Rym B, Amri M.2013. Anthropometric and Physical Characteristics of Tunisians Young Soccer PlayersJ. Advances in Physical Education. 2013; 3(3), 125-130

20. NiemanC. Exercise Testing and Prescription: A Health-Related Approach. 7th ed. New York, NY: McGraw-Hill Co, Inc; 2011: 102-116.

21. Reeves SL, Poh BK, Brown M, Tizzard NH, Ismail MN. Anthropometric measurements and body composition of English and Malaysian footballers. Malaysian Journal of Nutrition, 1999: 5, 79_86.

22. Reilly T, Bangsbo J, Franks A. Anthropometric and physiological predispositions for elite soccer. Journal of Sports Sciences, 2000: 18, 669-683.

23. Slavko R, Hilfiker R, Claiys P, Clijsen R, Taeytnansa J. Positionspecific and Team-ranking-related Morphological characteristics in German Amateur Soccer Players-A Descriptive Study- Anthropometry in Amateur Soccer players. International Journal of Applied Sports Sciences, 2011: 23, 8-182. 
24. Sinning WE, Dolny DG, Little,KD. "Validity of 'generalised' equations for body composition analysis in male athletes". Medicine and Science in Sports 1996;17: 124-130.

25. Stolen TK, Chamari C, Castagna U, Wisloff. Physiology of soccer: An update. J. Sports Med.2005;35: 501-536.

26. Stroyer J, Hansen L, Klausen K. Physiological profile and activity pattern of young soccer players during match play. Medicine Sciences Sports Exercises, 2004: 36, 168-174.
27. Singh S, Singh K, Singh M. Anthropometric measurements, body composition and somatotype of high jumpers. Brazilian Journal of Biochemistry, 2010; 4 (4): 266-271.

28. Verducci Frank M. Measurement and Concepts in Physical Education. St. Louis: the C. V. Mosby Company, 1980: 215. 